\title{
Energy dependence of identified hadron spectra and event-by-event fluctuations in $p+p$ interactions from NA61/SHINE at the CERN SPS
}

\author{
Maciej Rybczyński (for the NA61/SHINE Collaboration)* \\ Institute of Physics, Jan Kochanowski University, PL-25406 Kielce, Poland \\ E-mail: maciej.rybczynski@ujk.edu.pl
}

The NA61/SHINE Collaboration:

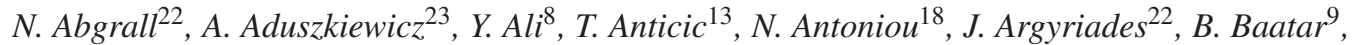

A. Blondel ${ }^{22}$, J. Blumer ${ }^{5}$, M. Bogomilov ${ }^{4}$, A. Bravar ${ }^{22}$, W. Brooks ${ }^{1}$, J. Brzychczyk $^{8}$, A. Bubak ${ }^{12}$

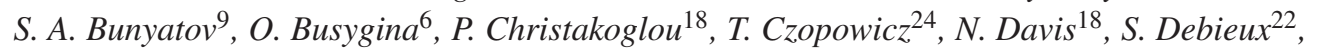

H. Dembinski ${ }^{5}$ F. Diakonos ${ }^{18}$, S. Di Luise ${ }^{2}$, W. Dominik ${ }^{23}$, T. Drozhzhova ${ }^{15}$, J. Dumarchez ${ }^{11}$,

K. Dynowski ${ }^{24}$, R. Engel $^{5}$, A. Ereditato ${ }^{20}$, L. Esposito ${ }^{2}$, G. A. Feofilov ${ }^{15}$, Z. Fodor ${ }^{10}$,

A. Ferrero ${ }^{22}$, A. Fulop ${ }^{10}$, M. Gaździcki ${ }^{17,21}$, M. Golubeva $^{6}$, B. Grabez $^{26}$, K. Grebieszkow ${ }^{24}$,

A. Grzeszczuk ${ }^{12}$, F. Guber ${ }^{6}$, H. Hakobyan ${ }^{1}$, T. Hasegawa ${ }^{7}$, M. Hierholzer ${ }^{20}$, R. Idczak ${ }^{25}$,

S. Igolkin ${ }^{15}$, Y. Ivanov ${ }^{1}$, A. Ivashkin ${ }^{6}$, D. Jokovic ${ }^{26}$, K. Kadija $^{13}$, A. Kapoyannis ${ }^{18}$,

N. Katrynska ${ }^{25}$, E. Kaptur ${ }^{12}$, D. Kielczewska ${ }^{23}$, D. Kikola ${ }^{24}$, M. Kirejczyk ${ }^{23}$, J. Kisiel ${ }^{12}$,

T. Kiss ${ }^{10}$, S. Kleinfelder ${ }^{27}$, T. Kobayashi ${ }^{7}$, V. I. Kolesnikov ${ }^{9}$, D. Kolev ${ }^{4}$, V. P. Kondratiev ${ }^{15}$,

A. Korzenev ${ }^{22}$, S. Kowalski ${ }^{12}$, A. Krasnoperov ${ }^{9}$, S. Kuleshov ${ }^{1}$, A. Kurepin ${ }^{6}$, D. Larsen $^{19}$,

A. Laszlo ${ }^{10}$, V. V. Lyubushkin ${ }^{9}$, M. Mackowiak-Pawlowska ${ }^{21,24}$, Z. Majka $^{8}$, B. Maksiak ${ }^{24}$,

A. I. Malakhov ${ }^{9}$, D. Maletic ${ }^{26}$, A. Marchionni ${ }^{2}$, A. Marcinek ${ }^{8}$, V.Marin ${ }^{6}$, K. Marton $^{10}$,

H.-J. Mathes ${ }^{5}$, T. Matulewicz ${ }^{23}$, V. Matveev ${ }^{6,9}$, G. L. Melkumov ${ }^{9}$, St. Mrówczyński ${ }^{17}$,

S. Murphy ${ }^{22}$, T. Nakadaira ${ }^{7}$, M. Nirkko ${ }^{20}$, K. Nishikawa ${ }^{7}$, T. Palczewski ${ }^{14}$, G. Palla ${ }^{10}$,

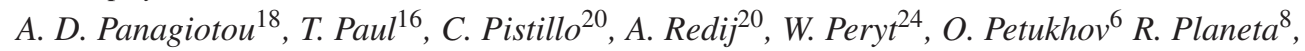

J. Pluta ${ }^{24}$, B. A. Popov ${ }^{9}$, M. Posiadala ${ }^{23}$,S. Puławski ${ }^{12}$, J. Puzovic ${ }^{26}$, W. Rauch ${ }^{3}$, M. Ravonel ${ }^{22}$, R. Renfordt ${ }^{21}$, A. Robert ${ }^{11}$, D. Röhrich ${ }^{19}$, E. Rondio ${ }^{14}$, M. Roth ${ }^{5}$, A. Rubbia ${ }^{2}$, A. Rustamov ${ }^{21}$, M. Rybczynski ${ }^{17}$, A. Sadovsky ${ }^{6}$, K. Sakashita ${ }^{7}$, M. Savic ${ }^{26}$, T. Sekiguchi ${ }^{7}$, P. Seyboth ${ }^{17}$,

M. Shibata ${ }^{7}$, R. Sipos ${ }^{10}$, E. Skrzypczak ${ }^{23}$, M. Slodkowski ${ }^{24}$, P. Staszel ${ }^{8}$, G. Stefanek ${ }^{17}$,

J. Stepaniak ${ }^{14}$, H. Stroebele ${ }^{21}$, T. Susa ${ }^{13}$, M. Szuba $^{5}$, M. Tada ${ }^{7}$, V. Tereshchenko ${ }^{9}$, T. Tolyhi ${ }^{10}$,

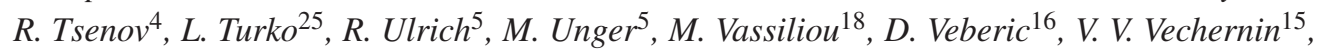

G. Vesztergombi ${ }^{10}$, L. Vinogradov ${ }^{15}$, A. Wilczek ${ }^{12}$, Z. Wlodarczyk ${ }^{17}$, A. Wojtaszek ${ }^{17}$,

O. Wyszyński ${ }^{8}$ L. Zambelli ${ }^{11}$ W. Zipper ${ }^{12}$ 


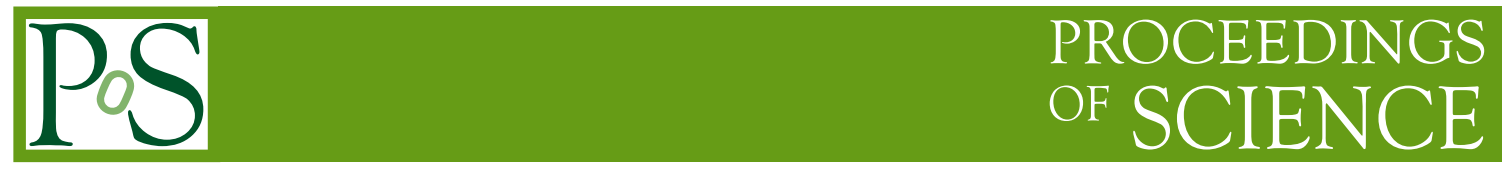

${ }^{1}$ The Universidad Tecnica Federico Santa Maria, Valparaiso, Chile

${ }^{2}$ ETH, Zurich, Switzerland

${ }^{3}$ Fachhochschule Frankfurt, Frankfurt, Germany

${ }^{4}$ Faculty of Physics, University of Sofia, Sofia, Bulgaria

${ }^{5}$ Karlsruhe Institute of Technology, Karlsruhe, Germany

${ }^{6}$ Institute for Nuclear Research, Moscow, Russia

${ }^{7}$ Institute for Particle and Nuclear Studies, KEK, Tsukuba, Japan

${ }^{8}$ Jagiellonian University, Cracow, Poland

${ }^{9}$ Joint Institute for Nuclear Research, Dubna, Russia

${ }^{10}$ Wigner Research Centre for Physics of the Hungarian Academy of Sciences, Budapest, Hungary

${ }^{11}$ LPNHE, University of Paris VI and VII, Paris, France

${ }^{12}$ University of Silesia, Katowice, Poland

${ }^{13}$ Rudjer Boskovic Institute, Zagreb, Croatia

${ }^{14}$ National Center for Nuclear Research, Warsaw, Poland

${ }^{15}$ St. Petersburg State University, St. Petersburg, Russia

${ }^{16}$ Laboratory of Astroparticle Physics, University Nova Gorica, Nova Gorica, Slovenia

${ }^{17}$ Jan Kochanowski University in Kielce, Poland

${ }^{18}$ University of Athens, Athens, Greece

${ }^{19}$ University of Bergen, Bergen, Norway

${ }^{20}$ University of Bern, Bern, Switzerland

${ }^{21}$ University of Frankfurt, Frankfurt, Germany

${ }^{22}$ University of Geneva, Geneva, Switzerland

${ }^{23}$ Faculty of Physics, University of Warsaw, Warsaw, Poland

${ }^{24}$ Warsaw University of Technology, Warsaw, Poland

${ }^{25}$ University of Wrocław, Wrocław, Poland

${ }^{26}$ University of Belgrade, Belgrade, Serbia

${ }^{27}$ University of California, Irvine, USA

NA61/SHINE at the CERN SPS is a fixed-target experiment pursuing a rich physics program including measurements for heavy ion, neutrino and cosmic ray physics. The main goal of the ion program is to explore the most interesting $T, m u_{B}$ region of the phase diagram of strongly interacting matter. We plan to study the properties of the onset of deconfinement and to search for the signatures of the critical point. The search is performed by varying collision energy (13A$158 \mathrm{~A} \mathrm{GeV/c}$ ) and system size (p+p, Be+Be, $\mathrm{Ar}+\mathrm{Ca}, \mathrm{Xe}+\mathrm{La})$.

Thanks to its large acceptance and excellent particle identification capability NA61/SHINE is well suited for performing high-precision particle production measurements as well as for studying event-by-event fluctuations in $\mathrm{p}+\mathrm{p}, \mathrm{p}+$ nucleus and nucleus+nucleus collisions.

Preliminary results on $\mathrm{p}+\mathrm{p}$ interactions at 20,31, 40, 80 and $158 \mathrm{GeV} / \mathrm{c}$ are presented. They include inclusive spectra of pi+, pi-, K- and protons as a function of transverse momentum/mass and rapidity as well as event-by-event fluctuations of transverse momentum, azimuthal angle and chemical composition. The new NA61 measurements are compared with the corresponding results of NA49 on central $\mathrm{Pb}+\mathrm{Pb}$ collisions and with predictions of Monte Carlo models.

Finally, the future plans of NA61/SHINE are summarised.

Xth Quark Confinement and the Hadron Spectrum,

October 8-12, 2012

TUM Campus Garching, Munich, Germany 


\section{Introduction}

NA61/SHINE (SPS Heavy Ion and Neutrino Experiment) is a fixed-target experiment operating since 2007 in the North Area of the CERN Super Proton Synchrotron, using a largeacceptance hadronic spectrometer to study a wide range of phenomena in a number of different hadron+hadron, hadron+nucleus and nucleus+nucleus reactions [1]. It is the successor of the NA49 experiment, which took data in the years 1994-2002, and reuses most of the NA49 hardware and software [2]. Large acceptance (around $50 \%$ for $p_{T} \leq 2.5 \mathrm{GeV} / \mathrm{c}$ ), high momentum resolution $\left(\sigma(p) / p^{2} \approx 10^{-4}(\mathrm{GeV} / \mathrm{c})^{-1}\right)$ and tracking efficiency (over $95 \%$ ), and excellent particle-identification capabilities $\left(\sigma\left(\frac{\mathrm{d} E}{\mathrm{~d} x}\right) / \frac{\mathrm{d} E}{\mathrm{~d} x} \approx 4 \%, \sigma\left(t_{T o F}\right) \approx 100 \mathrm{ps}\right)$ make it an excellent tool for investigating hadron spectra.

The ultimate goal is to explore the phase diagram of strongly interacting matter in collisions of different systems from $\mathrm{p}+\mathrm{p}$, through $\mathrm{p}+\mathrm{A}$, to $\mathrm{A}+\mathrm{A}$ collisions at projectile momenta of $13 \mathrm{~A}, 20 \mathrm{~A}$, $30 \mathrm{~A}, 40 \mathrm{~A}, 80 \mathrm{~A}$ and $158 \mathrm{~A} \mathrm{GeV/c}$. The energy scan of $\mathrm{p}+\mathrm{p}$ reactions was completed in 2009-2011 and preliminary results will be presented. Data on $\mathrm{Be}+\mathrm{Be}$ collisions at the three top energies were recorded in 2011, the remaining energies will be taken in 2012. The energy scan of $\mathrm{p}+\mathrm{Pb}, \mathrm{Ar}+\mathrm{Ca}$ and $\mathrm{Xe}+\mathrm{La}$ collisions is foreseen until 2016. In addition a beam energy scan of $\mathrm{Pb}+\mathrm{Pb}$ collisions is planned. The explored energy range probes an important region in the phase diagram of strongly interacting matter. Indeed, the NA49 collaboration reported signals for the onset of deconfinement encoded in nonmonotonic behavior of excitations functions of several hadronic observables [3-5]. Moreover, the critical point at the location predicted by several theory groups [6] can be probed at SPS energies.

\section{Detector Set-up}

Figure 1 shows a diagram of the NA61/SHINE apparatus. The primary tracking detectors are four large-volume Time Projection Chambers (TPCs) inherited from NA49. Two of them (Vertex TPCs) are located inside superconducting magnets along the beam line, the other two (Main TPCs) are placed symmetrically to the beam line further downstream. Another, smaller chamber (Gap TPC) is placed between the VTPCs. Together, these chambers measure trajectories and momenta of particles and allow their identification through ionisation energy loss.

Three Time-of-Flight (ToF) walls are positioned just downstream of the MTPCs, two on the sides inherited from NA49 and the central one added by NA61 in 2007. Measurements from these detectors complement particle identification in the momentum range where $\mathrm{d} E / \mathrm{d} x$ alone is ambiguous.

The most downstream detector of the apparatus is the Projectile Spectator Detector (PSD), installed in 2011. The purpose of this calorimeter is to provide precise, high-granularity event-byevent measurements of the energy of non-interacting fragments of the projectile nuclei, making it possible to determine the centrality of the collision and the orientation of the reaction plane.

Finally, a number of additional detectors installed in the beam line both upstream and downstream of the target monitor beam properties and provide trigger information.

\footnotetext{
*Speaker.
} 


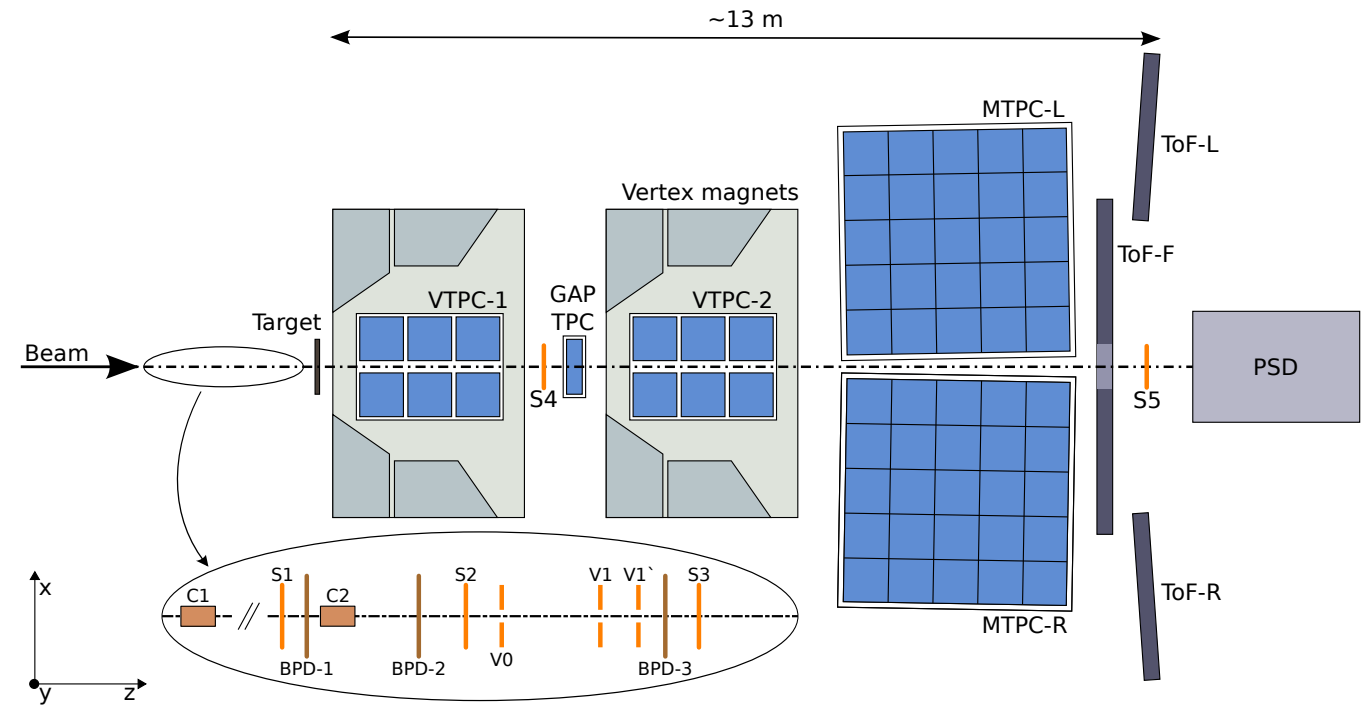

Figure 1: (Color online) Layout of the NA61/SHINE experimental set-up (top view, not to scale).For details see [2].

A sophisticated beam line set-up was designed and put in place by the CERN acceleratorsand-beams department in collaboration with NA61 for the purpose of providing beryllium beams for SHINE from the fragmentation of $\mathrm{Pb}$ ions. At the moment the only primary beams the SPS can provide are protons and lead ions, with argon and xenon to be made possible during the long shutdown of the accelerator complex in 2013-2014. As adding new species of primary beams is an immensely complicated task, beryllium beams for SHINE were instead produced in a fragmentation beam line. Primary $\mathrm{Pb}$ ions from the SPS were directed onto a beryllium block far upstream in the beam line and the resulting fragments of the projectile were guided through a sequence of magnets, collimators, degraders and beam detectors in order to filter out beryllium ions. This configuration was proven to provide highly pure ${ }^{7} \mathrm{Be}$ ions over a wide range of Pb-beam energies.

\section{Results}

Data taking for the NA61/SHINE ion program started with the energy scan of $p+p$ interactions. These data are needed to establish a base line reference for the results on collisions of light and medium size nuclei. The latter are particularly important in the search for the critical point of strongly interacting matter.

\subsection{Inclusive particle spectra in $p+p$ interactions}

First measurements of $\pi^{-}$spectra in $\mathrm{p}+\mathrm{p}$ collisions at $20,31,40,80$, and $158 \mathrm{GeV} / \mathrm{c}$ are available. Figure 2 (left) presents the transverse mass $\left(m_{T}\right)$ spectra at mid-rapidity for negatively charged pions in inelastic $p+p$ collisions. The results are corrected for reconstruction inefficiencies, acceptance, and feed-down using Monte Carlo simulations. Figure 2 (right) shows the ratios of $m_{T}$ spectra from central $(7 \%) \mathrm{Pb}+\mathrm{Pb}$ to inelastic $\mathrm{p}+\mathrm{p}$ collisions $[3,4]$. The observed differences do not change with collision energy and could be due to transverse collective flow present in $\mathrm{Pb}+\mathrm{Pb}$. 

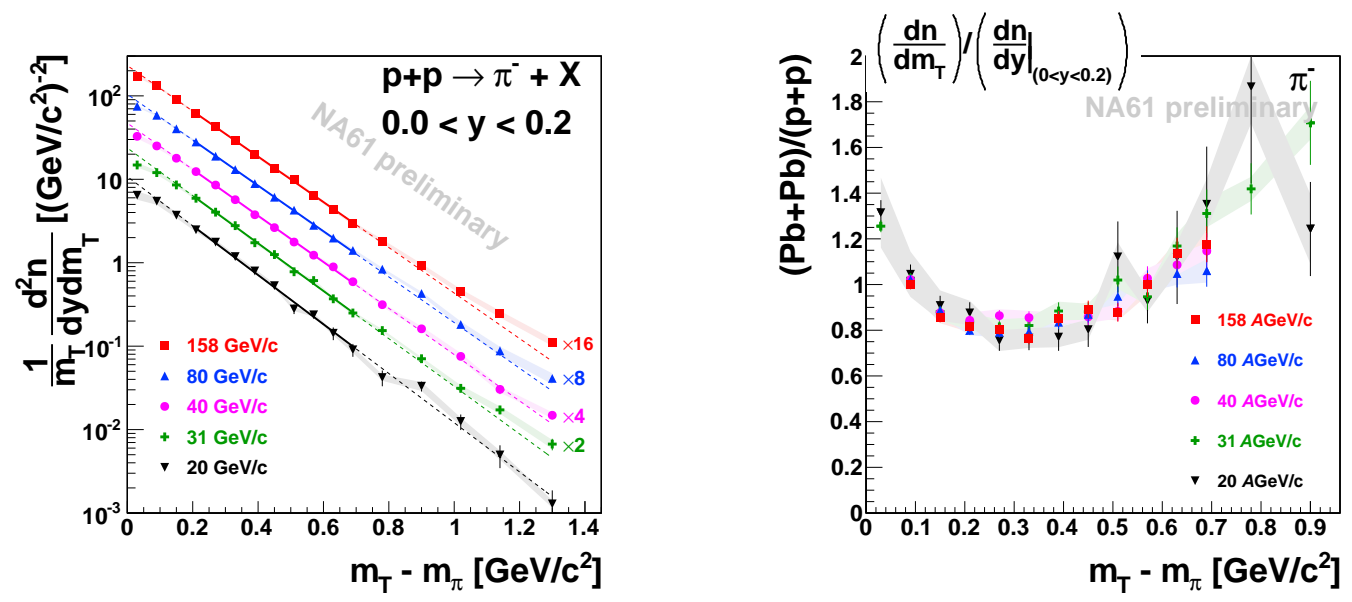

Figure 2: (Color online) Left: Transverse-mass spectra at mid-rapidity for negatively charged pions fitted with an exponential function in the $0.2<m_{T}-m_{\pi}<0.7 \mathrm{GeV} / \mathrm{c}^{2}$ interval. Right: The ratio of $\mathrm{Pb}+\mathrm{Pb}$ to $\mathrm{p}+\mathrm{p}$ transverse mass spectra. Results refer to inelastic $\mathrm{p}+\mathrm{p}$ interactions at $20-158 \mathrm{GeV} / \mathrm{c}$.

The missing part of the $m_{T}$ spectra are extrapolated by fitting the distributions with an exponential function. This allows to obtain the $m_{T}$ integrated rapidity spectra depicted in Figure 3 (left). The spectra are well described by a sum of two symmetrically displaced Gaussian functions. When compared to $\mathrm{Pb}+\mathrm{Pb}$ data the widths of $\pi^{-}$rapidity distributions for $\mathrm{p}+\mathrm{p}$ are clearly narrower, see Figure 3 (right). The possible reasons are isospin effects and/or longitudinal collective flow in central $\mathrm{Pb}+\mathrm{Pb}$ collisions. The two-Gaussian fit allows to extrapolate the rapidity spectrum and calculate the mean $\pi^{-}$multiplicity.

Figure 4 (left) shows that the NA61/SHINE pion multiplicities are in good agreement with the world data. Figure 4 (right) presents the "kink" plot, showing the total pion multiplicity normalized to the number of wounded nucleons versus the Fermi variable $F \approx s^{0.25}$. Unlike for $\mathrm{p}+\mathrm{p}$ interactions, a change of slope is visible around $30 \mathrm{~A} \mathrm{GeV}$ in central $\mathrm{Pb}+\mathrm{Pb}$ and $\mathrm{Au}+\mathrm{Au}$ collisions. Such an increase can be explained by an increase of the effective number of degrees of freedom when going from hadron gas to QGP as a consequence of the activation of partonic degrees of freedom. Also, preliminary results on $p_{T}$ and rapidity spectra of charged pions, negatively charged kaons, and protons were obtained in inelastic p+p interactions at 40,80 and $158 \mathrm{GeV} / \mathrm{c}$. These will provide the baseline for the study of the properties of the onset of deconfinement by looking for the kink, horn, step and dale structures [7-9] in collisions of light and intermediate mass nuclei $(\mathrm{Be}+\mathrm{Be}, \mathrm{Ar}+\mathrm{Ca}, \mathrm{Xe}+\mathrm{La})$.

\subsection{Event by Event fluctuations in $\mathrm{Pb}+\mathrm{Pb}$ and $\mathrm{p}+\mathrm{p}$ collisions}

The value of $\mathrm{d} E / \mathrm{d} x$ does not allow to identify each particle uniquely as the $\mathrm{d} E / \mathrm{d} x$ distributions overlap. The identity method [10] was developed to extract second and third moments (pure and mixed) of identified particle multiplicity distribution corrected for this imperfect identification. First fluctuation measurements in $\mathrm{p}+\mathrm{p}$ give a unique opportunity to compare $\mathrm{Pb}+\mathrm{Pb}$ and $\mathrm{p}+\mathrm{p}$ results at the SPS energies. 

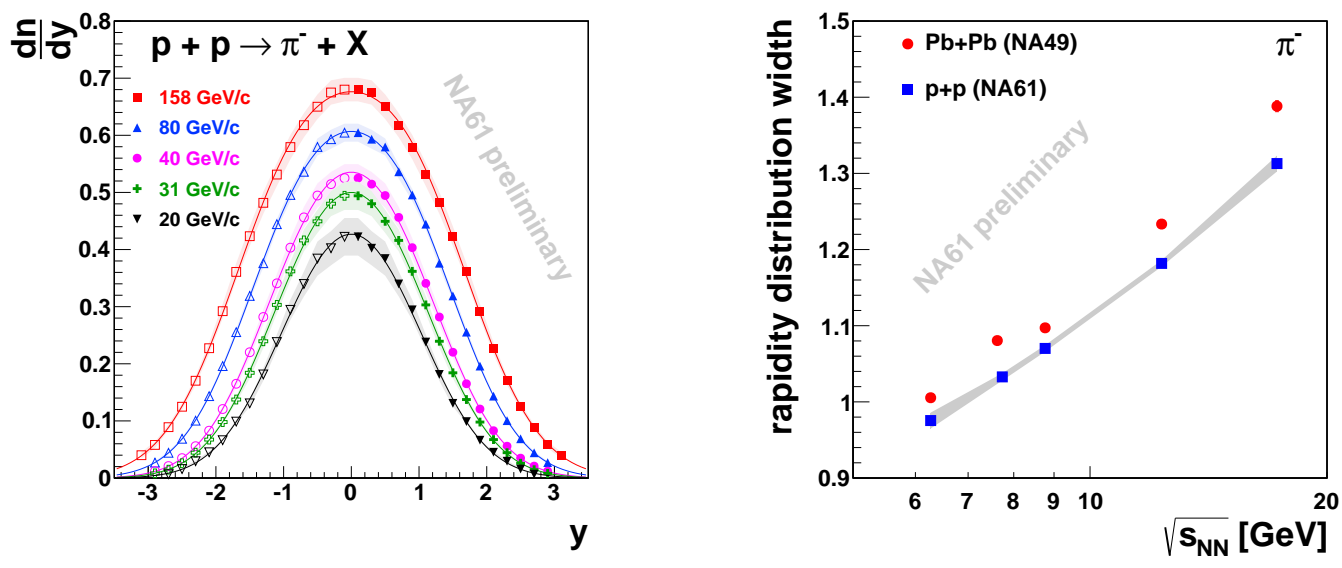

Figure 3: (Color online) Left: Rapidity spectra of negatively charged pions fitted with the sum of two Gaussian functions. Right: The width of rapidity spectra of $\pi^{-}$produced in p+p interactions at $20-158 \mathrm{GeV} / \mathrm{c}$ compared to NA49 results from central $\mathrm{Pb}+\mathrm{Pb}$ collisions $[3,4]$.
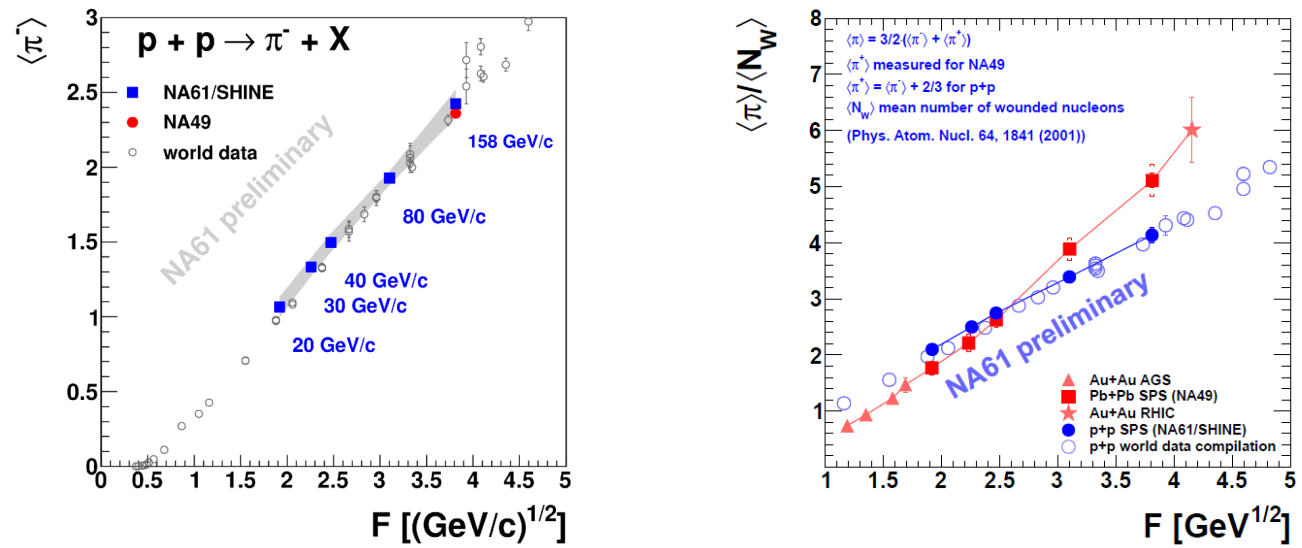

Figure 4: (Color online) Left: Mean negatively charged pion multiplicity. Right: Mean pion multiplicity per participant nucleon. Both are shown as function of the Fermi variable $F \approx s^{0.25}$.

Preliminary results on multiplicity fluctuations of $\pi=\pi^{+}+\pi^{-}, K=K^{+}+K^{-}$, and $p=p+\bar{p}$ were obtained for $\mathrm{p}+\mathrm{p}$ interactions at $31,40,80$ and $158 \mathrm{GeV} / \mathrm{c}$. From the first and second corrected moments of the multiplicity distributions, $N_{i}$ and $N_{i}^{2},(i=\pi, K, p)$, the scaled variance

$$
\omega_{i}=\frac{\left\langle N_{i}^{2}\right\rangle-\left\langle N_{i}\right\rangle^{2}}{\left\langle N_{i}\right\rangle}
$$

was computed. For Poisson multiplicity distributions $\omega_{i}=1$ and does not depend on the number of wounded nucleons. However, it is sensitive to the fluctuations of the wounded nucleon number. This may distort the comparison of $\mathrm{p}+\mathrm{p}$ data with results from nucleus+nucleus collisions.

All studied scaled variances increase with increasing collision energy (Figure 5). For kaons 
$\omega>1$ for higher energies in agreement with predictions of the EPOS model. This may be caused by the correlation in $K^{+}$and $K^{-}$production due to strangeness conservation. For protons $\omega$ is below 1, probably related to baryon number conservation. A comparison of NA61/SHINE results on multiplicity fluctuations (i.e. $\omega_{\pi}$ ) with the magnitude of fluctuations expected at the critical point [11] suggests that the systematic and statistical errors are small enough for a sensitive search of the critical point in NA61/SHINE.

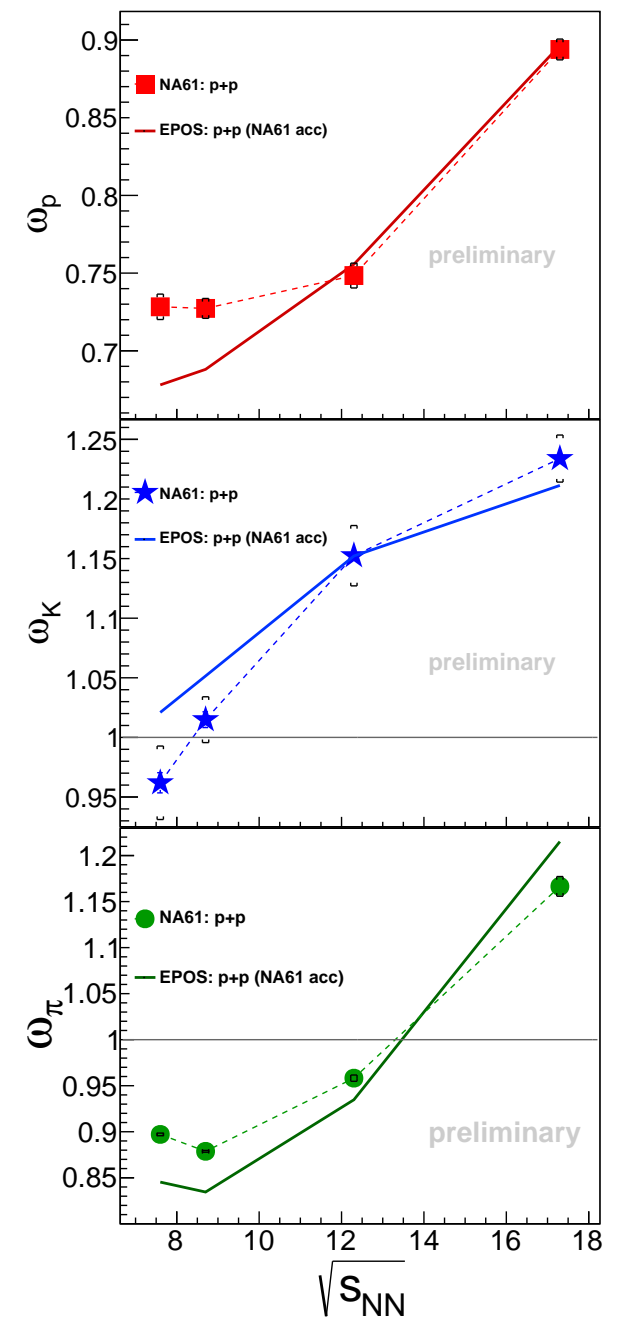

Figure 5: (Color online) Scaled variance for $p+\bar{p}$ (upper panel), $K^{+}+K^{-}$(middle panel) and $\pi^{+}+\pi^{-}$ (bottom panel) multiplicity distributions for $\mathrm{p}+\mathrm{p}$ collisions as a function of center of mass energy. The NA61/SHINE results are shown by filled symbols connected by dashed lines and compared to EPOS model calculations depicted by a solid lines.

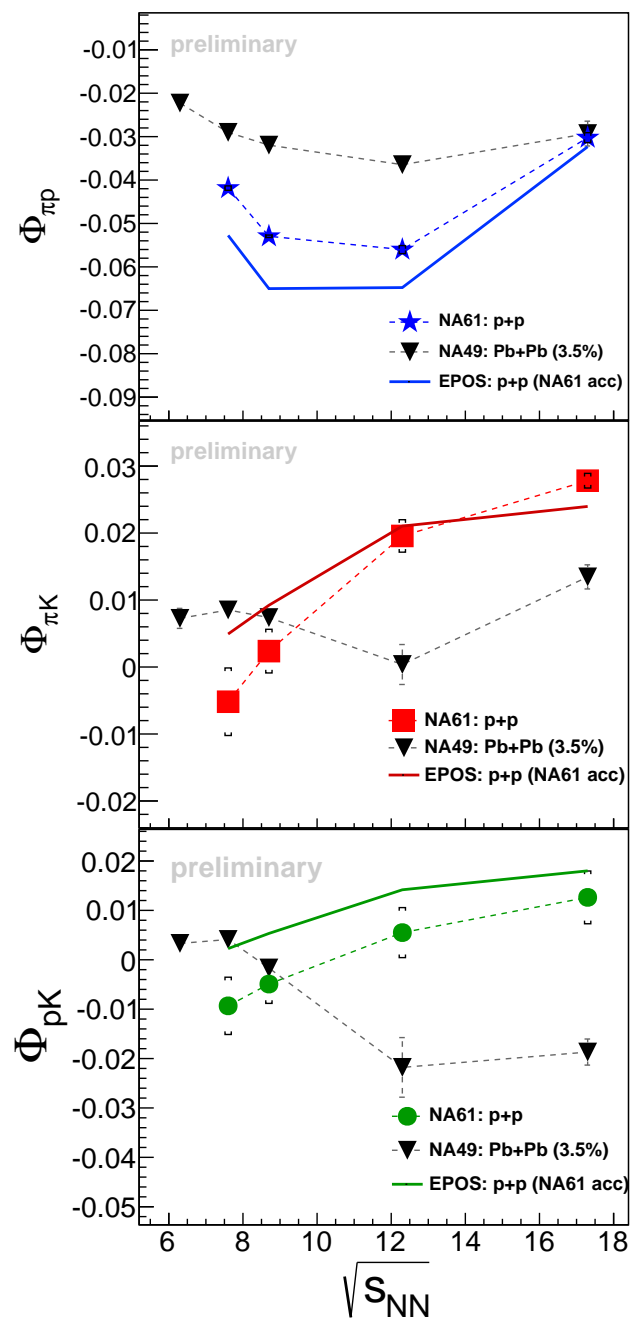

Figure 6: (Color online) $\Phi_{i j}$ measure as a function of center of mass energy for $\mathrm{p}+\mathrm{p}$ (NA61/SHINE) and central $\mathrm{Pb}+\mathrm{Pb}$ (NA49) collisions. The $\mathrm{p}+\mathrm{p}$ data are compared with the EPOS model predictions (solid lines).

In order to compare results for $\mathrm{p}+\mathrm{p}$ and central $\mathrm{Pb}+\mathrm{Pb}$ collisions, the strongly intensive measure $\Phi_{i j}[12,13]$ defined for two hadron types, $i$ and $j$, was chosen. It is defined as: 


$$
\Phi_{i j}=\frac{\sqrt{\left\langle N_{i}\right\rangle\left\langle N_{j}\right\rangle}}{\left\langle N_{i}+N_{j}\right\rangle} \cdot\left(\sqrt{\Sigma^{i j}}-1\right)
$$

where $\Sigma^{i j}=\left\langle N_{i}\right\rangle \omega_{j}+\left\langle N_{j}\right\rangle \omega_{i}+2 \cdot\left(\left\langle N_{i j}\right\rangle-\left\langle N_{i}\right\rangle\left\langle N_{j}\right\rangle\right) \cdot\left\langle N_{i}+N_{j}\right\rangle$. As a strongly intensive measure, $\Phi_{i j}$ is not only independent of number of wounded nucleons or volume but also of their fluctuations. Figure 6 shows the energy dependence of $\Phi_{i j}$ for combinations of two hadron types: $\pi p, \pi K$, and $p K$. When no inter-particle correlations are present $\Phi_{i j}=0$. For $\pi K$ and $p K$ the values of $\Phi_{i j}$ increase with increasing energy. There is a minimum for $\Phi_{\pi p}$ between 7.3 and $8.7 \mathrm{GeV}$. A similar but weaker effect is visible in $\mathrm{Pb}+\mathrm{Pb}$ interactions. It also appears in the EPOS model. The increase of $\Phi_{\pi K}$ for $\mathrm{p}+\mathrm{p}$ interactions is not visible in $\mathrm{Pb}+\mathrm{Pb}$ collisions. $\Phi_{p K}$ shows a clear difference between results for $\mathrm{p}+\mathrm{p}$, which increase with increasing energy, and for $\mathrm{Pb}+\mathrm{Pb}$ which decrease with increasing energy. Both dependences cross zero at the same energy $\sqrt{s_{N N}} \approx 8.7 \mathrm{GeV}$.

\section{Summary}

We presented preliminary results from NA61/SHINE on particle production in inelastic $\mathrm{p}+\mathrm{p}$ collisions. These data represent the first phase of the scan of the phase diagram of strongly interacting matter. Inclusive particle spectra and mean multiplicities were obtained. Event-by-event chemical fluctuations were analyzed using a novel approach, the Identity Method. These results will serve as an important reference for $\mathrm{A}+\mathrm{A}$ collisions.

This work was supported by the Polish Ministry of Science and Higher Education under the grant 667/N-CERN/2010/0.

\section{References}

[1] N. Antoniou et al. [NA61/SHINE Collaboration], CERN-SPSC-2006-034, CERN-SPSC-P-330 (2006)

[2] S. Afanasev et al. [NA49 Collaboration, Nucl.Instrum.Meth. A 430, 210 (1999)

[3] S. V. Afanasiev et al. [NA49 Collaboration], Phys. Rev. C 66, 054902 (2002) [nucl-ex/0205002].

[4] C. Alt et al. [NA49 Collaboration], Phys. Rev. C 77, 024903 (2008) [arXiv:0710.0118 [nucl-ex]].

[5] C. Alt et al. [NA49 Collaboration], Phys. Rev. C 75, 064904 (2007) [nucl-ex/0612010].

[6] Z. Fodor and S. D. Katz, JHEP 0404, 050 (2004) [hep-lat/0402006].

[7] M. Gazdzicki and M. I. Gorenstein, Acta Phys. Polon. B 30, 2705 (1999) [hep-ph/9803462].

[8] T. Anticic et al. [NA49 Collaboration], Eur. Phys. J. C 65, 9 (2010) [arXiv:0904.2708 [hep-ex]].

[9] M. Gazdzicki, M. Gorenstein and P. Seyboth, Acta Phys. Polon. B 42, 307 (2011) [arXiv:1006.1765 [hep-ph]].

[10] A. Rustamov and M. I. Gorenstein, Phys. Rev. C 86, 044906 (2012) [arXiv:1204.6632 [nucl-th]].

[11] K. Grebieszkow [NA49 Collaboration], Nucl. Phys. A 830, 547C (2009) [arXiv:0907.4101 [nucl-ex]].

[12] M. I. Gorenstein and M. Gazdzicki, Phys. Rev. C 84, 014904 (2011) [arXiv:1101.4865 [nucl-th]].

[13] M. Gazdzicki, Eur. Phys. J. C 8, 131 (1999) [nucl-th/9712050]. 